\title{
A Study of the Formation of Velocity Counter Lines and Secondary (Spiral) Flows in Different Cross Sections of Different Arcs
}

\author{
Ali Liaghat \\ Shiraz Branch \\ Islamic Azad University \\ Shiraz, Iran
}

\author{
Nima Tavanpour \\ Shiraz Branch \\ Islamic Azad University \\ Shiraz, Iran
}

\begin{abstract}
The mechanical properties of flow are very complex in channel arcs. Therefore, dynamic numerical models of fluids are considered effective tools in predicting such flow fields. In this study, the numerical model was validated by the measures of a uniform U-shaped arc with a width of 0.6 meter. Then two similar $U$ shaped arcs, divergent and convergent, were simulated by a three-dimensional numerical model with variable widths from 0.6 to 0.75 meters and 0.6 to 0.45 meters. Validating the numerical model by measured data in the uniform 180-degree arc showed that the model can simulate the flow field in the uniform arc very well. Results regarding several parameters such as rout of maximum velocity, maximum velocity line, water level variations, power of spiral flow, existence of a rotating cell are stated and discussed.
\end{abstract}

Keywords-Arc; Secondary flow (spiral); Velocity Counters; SSIIM three-dimensional numerical model

\section{INTRODUCTION}

Simulation of flow patterns is the most interesting subject in river engineering and sedimentation studies [1]. Predicting rivers' behavior on bends is obviously the focus of interest, as most rivers have meandrous form. For meandrous rivers, flow patterns are too complex as flow mechanics has different characteristics on bends compared to straight channels. In general, forces influencing flows at a bend include centrifugal force due to Non-uniformity of vertical velocity profile, and combine with flow curve, cross-sectional stresses, and radius gradient of pressure caused by lateral slope of water surface [2]. Synchronous effects of such forces create a flow called Helical Flow. Many studies have been performed to investigate the flow characteristics and patterns in $180^{\circ}$ bends with solid beds, of which [3] is the earliest, but substantially criticized for setting the radial velocity component at zero. Rozovskii [4] was among the first researchers who performed comprehensive studies in this field. The most important result Rozovskii obtained was that the velocity distribution belongs to a 3-D flow, and that the velocity component exists in radius direction. Mosonyi and Gotz [5] were the first who paid attention to the manner of distribution of helical flow strength and its changes along channel. They showed that secondary flow can be well described by its strength changes. They reported the presence of second cycle of secondary flow near internal bend, which only occurs at $\mathrm{B} / \mathrm{h}<10$ ratios. Leschziner and Rodi [6] presented their 3-D numerical model by using the finite differences method. The most important result these researchers reported was the effect of lengthwise pressure gradient on flow pattern in swift bends. In [7], authors exercised the effect of secondary flow on depth-averaged equations by using stress diffusion matrix and studied flow pattern in $180^{\circ}$ bend with rigid bed through their 2-D numerical model. Their research indicated the effect of secondary flow on the origin of maximum velocity along channel. Booij modeled the structure of secondary flow in $180^{\circ}$ bend by using large eddy simulation method [8]. The most important point of his research was that disturbance turbulence $k-\varepsilon$ model fails to model the rotation against direction of secondary flow near external wall. In [9], authors did extensive research in this field. In [10], authors developed a 2-D model to compute the diffusion term in convection equation of suspended sediments, which increase efficiency of 2-D models to simulate the secondary flows in channel bends. They studied the over-time bed evolution in $180^{\circ}$ river bends with SSIIM 3-D model [10]. In [11], authors developed a 2-D numerical model which describes the hydrodynamic, sediment transportation and bed changes in river bends. In [12], authors investigated the flow characteristics such as effect of secondary flows on velocity distribution and path of longitudinal maximum velocity in $90^{\circ}$ bends with constant width and solid bed. In their research several parameters (e.g., stream wise and vertical velocity profile, bed shear stress distribution, stream wise and span wise slopes of water surface and helical flow strength) were compared in $180^{\circ}$ divergent and uniform bends.

\section{MATHEMATICAL MODEL AND METHODS}

Navier-Stokes equations for viscous and turbulent noncompressible fluid flows include continuity equation (1) and momentum conservation equation (2) are written as follows [1]:

$$
\frac{d U_{i}}{d x_{i}}=0 \quad(i=1,2,3)
$$




$$
\frac{\partial U_{i}}{\partial t}+U_{j} \frac{\partial U_{i}}{\partial x_{j}}=\frac{1}{\rho} \frac{\partial}{\partial x_{j}}\left(-P \delta_{i j}-\rho \overline{u_{i} u_{j}}\right), j=1,2,3
$$

Where $U_{i}$ is the average velocity component in $x_{i}$ direction; $\rho$ is the density of water; $P$ is pressure; $\delta_{i j}$ is the Kroncker delta, which is 1 if $\mathrm{i}=\mathrm{j}$ and 0 otherwise; and $\mathrm{x}_{\mathrm{i}}$ and $\mathrm{x}_{\mathrm{j}}$ are the general space dimensions. The last term in (2) is Reynolds stress, which represents the transport of momentum that can be attributed to turbulence. The Reynolds stress is often modeled with the Boussinesque approximation:

$$
\rho \overline{u_{i} u_{j}}=\rho v_{t}\left(\frac{\delta U_{i}}{\partial x_{j}}+\frac{\delta U_{j}}{\partial x_{i}}\right)-\frac{2}{3} \rho \cdot k \cdot \delta_{i j}
$$

Where $u_{t}$ is eddy - viscosity $k-\varepsilon$ model calculates the eddy viscosity as

$$
v_{t}=C_{\mu}^{\prime} \frac{k^{2}}{\varepsilon}
$$

$k$ is turbulent kinetic energy defined by

$$
k=\frac{1}{2} \overline{u_{i} u_{j}}
$$

$k$ is modeled as

$$
\frac{\partial k}{\partial t}+U_{i} \frac{\partial k}{\partial x_{i}}=\frac{\partial}{\partial x_{i}}\left[\left(\frac{v_{t}}{\sigma_{k}}\right) \frac{\partial k}{\partial x_{j}}\right]+P_{k}-\varepsilon
$$

Where $P_{k}$ is given by

$$
P_{k}=v_{t} \frac{\partial U_{i}}{\partial x_{j}}\left(\frac{\partial U_{i}}{\partial x_{j}}+\frac{\partial U_{j}}{\partial x_{i}}\right)
$$

The dissipation of $k$ is denoted $\varepsilon$, and modeled as

$$
\frac{\partial \varepsilon}{\partial t}+U_{i} \frac{\partial \varepsilon}{\partial x_{i}}=\frac{\partial}{\partial x_{i}}\left[\left(\frac{\nu_{t}}{\sigma_{\varepsilon}}\right) \frac{\partial \varepsilon}{\partial x_{j}}\right]+C_{\varepsilon 1} \frac{\varepsilon}{k} P_{k}-C_{\varepsilon 2} \frac{\varepsilon^{2}}{k}
$$

In (4) through (8), $C_{\mu}, \sigma_{k}, \sigma_{\varepsilon}, C_{\varepsilon 1}$ and $C_{\varepsilon 2}$ are empirical constants, which were determined experimentally to be 0.09 , $1.0,1.3,1.44$, and 1.92 , respectively [13]. Boundary conditions for the Navier-Stokes equation (e.g., boundary condition for inflow, outflow, the water surface and the bed/wall) are similar to the diffusion-convection equation. Dirichlet boundary conditions have to be given at the inflow boundary, which is relatively straightforward for velocity. Usually it is more difficult to specify the turbulence. To specify the eddy viscosity, it is possible to estimate the shear stress $(\tau)$ at the entrance bed using a given velocity. Then, the turbulent kinetic energy $k$ at the entrance bed is determined by:

$$
k=\frac{\tau}{\rho \sqrt{c_{\mu}^{\prime}}}
$$

With the eddy viscosity $u_{t}$ and turbulent kinetic energy $k$ at the bed, (4) gives the value of $\varepsilon$ at the bed. If $k$ is assumed to vary linearly from the bed to the surface, then (4), together with the profile of the eddy viscosity, can be used to calculate the vertical distribution of $\varepsilon$. A zero gradient condition was used for outflow boundary. The free surface is computed using a fixed-lid approach, with zero gradients for all variables. The location of fixed lid and its movement as a function of time and the water flow field are computed by pressure and Bernoulli algorithm. The algorithm is based on pressure field. It uses the Bernoulli equation along the water surface to compute the water surface location based on fixed point downstream of bend in this study.

The wall law for rough boundaries [14] was used as a boundary condition for bed and wall:

$$
\frac{U}{U^{*}}=\frac{1}{\kappa} \ln \left(\frac{30 y}{k_{s}}\right)
$$

Where $U^{*}$ is the shear velocity, $U$ is the velocity at the center of the grid cell closest to the bed, $\kappa$ is a constant equal to $0.4, y$ is the distance from the wall to the center of the grid cell, and $k_{s}$ is wall roughness.

\section{RESULTS AND DISCUSSION}

\section{A. Verification of 3-D numerical model}

The experimental data is extract from a channel with $180^{\circ}$ bend used in Pirestani's lab studies [15]. Figure 1 show convergent and divergent $180^{\circ}$ bends studied in this paper, which has a mesh size $91 \times 91 \times 7$ in longitudinal, transverse and vertical direction, respectively. Mentioned bend has two straight paths, upstream and downstream, which were $7.2 \mathrm{~m}$ and $5.2 \mathrm{~m}$ long, respectively. Its wall and bed is made of Plexiglas with wall roughness of $0.0001 \mathrm{~m}\left(k_{s}=0.0001 \mathrm{~m}\right)$. The flow pattern was studied at a flow rate of $30 \mathrm{~L} / \mathrm{s}$ and a water depth of $0.15 \mathrm{~m}$ at the channel entrance, and, in order to verify numerical modeling, the results from modeling in the bend with uniform $0.6 \mathrm{~m}$ width were compared with Pirestanie's lab results [14], as shown in Figures 2 to 4 . It should be noted that in Pirestani's research, flow velocities were measured at different depths in 91 cross sections along the bend with a two dimensional portable emissions measurement systems (PEMS).
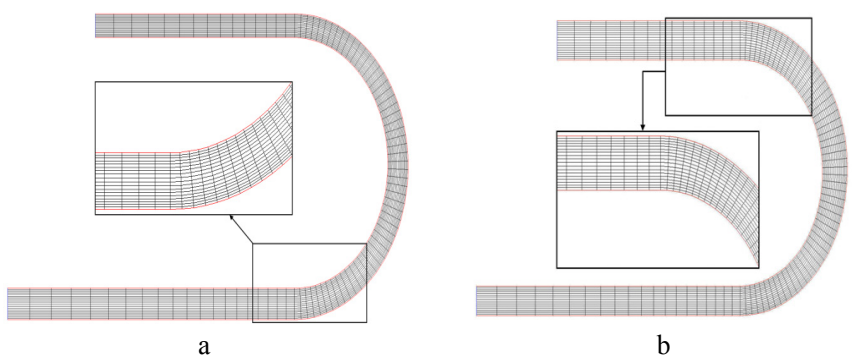

Fig. 1. Grid sample used in this study (a) Convergent bend and (b) Divergent bend

To find the optimal mesh size, grid independency investigations have performed. So that the mesh size has changed from $71 \times 12 \times 7$ in longitudinal, transverse and vertical direction, respectively as a coarse mesh to finest mesh size $351 \times 35 \times 15$. By comparison of maximum and minimum velocities, pressure and turbulent kinetic energy in different mesh finally the mesh size $91 \times 91 \times 7$ was selected as the optimal 
mesh size. Power low and second order upwind methods for discretization of convectional term were used. The result showed no significant difference between the velocity profiles calculated by two methods. There for in continue the power low method used.

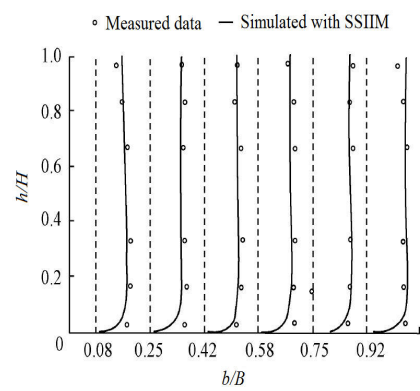

Fig. 2. Comparison of vertical velocity profile (continuous line) with measurement data (points) in $180^{\circ}$ cross section.

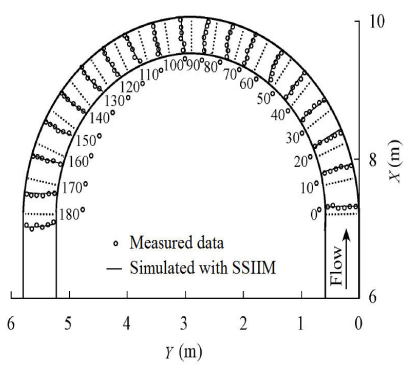

Fig. 3. Comparison of velocity profiles profile (continuous line) at the plane near water surface $(\mathrm{h}=0.145 \mathrm{~m}) \quad$ in different cross sections with lab data (points).

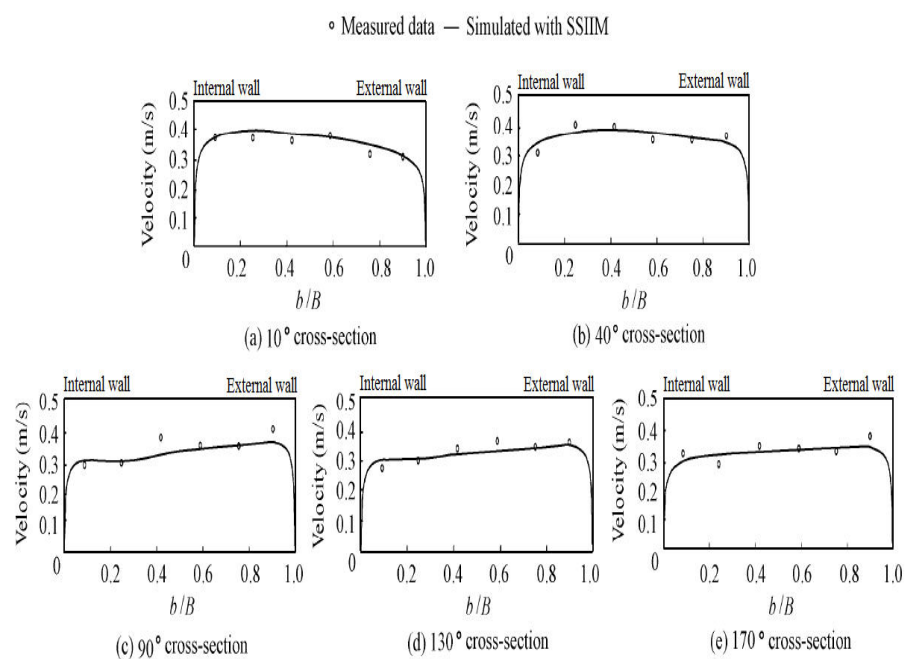

Fig. 4. Comparison of velocity profiles profile (continuous line) at the plane near water surface $(\mathrm{h}=0.145 \mathrm{~m})$ in different cross sections with lab data (points).

Figures 2 through 4 indicate that velocity profiles calculated by numerical modeling are in complete agreement with labmeasured data. Statistical indicators (e.g., ERMS and EM) were used to compare values of calculated and measured velocities at a plane of $\mathrm{h}=0.145 \mathrm{~m}$ :

$$
E_{R M S}=\sqrt{\frac{1}{N} \sum_{i=1}^{N}\left[V_{M i}-V_{P i}\right]^{2}}
$$

Where ERMS is root mean square of error, EM is mean error, $\mathrm{N}$ is number of measurement data, $V_{M i}$ and $V_{P i}$ are the values of the ith measured and calculated velocities, respectively. Results of statistical comparison are presented in Table I. The low values of ERMS and EM in the table indicate numerical model simulates well flow field in uniform bend.
TABLE I. STATISTICAL INDICATOR BETWEEN CALCULATED AND MEASURED VELOCITIES AT PLAN NEAR WATER SURFACE

\begin{tabular}{|c|c|c|c|c|c|}
\hline & \multicolumn{5}{|c|}{ Cross section } \\
\hline $\begin{array}{c}\text { Statistical } \\
\text { indicator }\end{array}$ & $10^{\circ}$ & $40^{\circ}$ & $90^{\circ}$ & $130^{\circ}$ & $170^{\circ}$ \\
\hline $\boldsymbol{E R M S ~ ( m / s ) ~}$ & 0.074 & 0.042 & 0.048 & 0.043 & 0.042 \\
\hline $\boldsymbol{E M}(\mathbf{m} / \mathbf{s})$ & -0.066 & -0.009 & 0.018 & 0.015 & 0.012 \\
\hline
\end{tabular}

\section{B. Comparison of vertical velocity profiles}

In this paper, the results from a convergent channel bend with a width ranging from $0.6 \mathrm{~m}$ at the beginning of the bend to $0.45 \mathrm{~m}$ at the end of the bend, divergent channel bend with a width ranging from $0.6 \mathrm{~m}$ to $0.75 \mathrm{~m}$ were compared with those of a channel with uniform $0.6 \mathrm{~m}$ width in order to compare flow characteristics of uniform channels with divergent and convergent ones. Vertical velocity profiles were taken into account on two cross sections, i.e. sections $45^{\circ}, 135^{\circ}$ (Figure 5). At both cross sections vertical velocity profiles show lower values in divergent bend than in uniform and convergent one, because velocity will decrease in divergent bend due to bend width bend expanding and we have opposite of this situation in convergent bend. Also, maximum and minimum of vertical velocity profile is seen at dimensionless distance of $0.75 \mathrm{~m}$ and $0.08 \mathrm{~m}$ from internal wall of channel. Additionally, vertical velocity profiles at $45^{\circ}$ cross section are close together for the three types of the bends, while at $135^{\circ}$ cross section the difference between them is more significant.

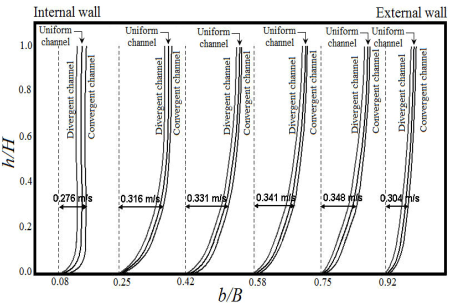

(a) $45^{\circ}$ cross-section

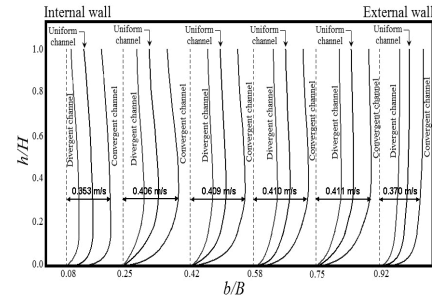

(b) $135^{\circ}$ cross-section
Fig. 5. Comparison of vertical velocity profiles at different cross sections of uniform, divergent and convergent bends.

\section{Comparison of lengthwise velocity Profiles in the bends}

Figure 6 shows the trends of changes in lengthwise velocity profile at the plane near water surface for three types of bend channels. In all cross sections the values of velocity in divergent bend shows slower velocity than in the uniform bend and the uniform bend velocity is slower than convergent bend due to bend expanding and decreasing in discharge per unit width. This difference will increase with the distance from the beginning of the bend. Also, lengthwise velocity profile have the same shape in three type of bends which shows that the maximum lengthwise velocity occurs near the internal wall at the beginning of the bend and after entering the bend, it migrates gradually toward the external wall.

Table II discuses about comparison of differences between values of velocities in uniform and divergent bends and also uniform and Convergent bends. In all cross sections we see more decreasing of lengthwise velocity in internal bend rather 
than external bend. This more decreasing of lengthwise velocity in internal bend rather than external bend is almost constant and about of $5 \%$ to $7 \%$ and with pasting from beginning of bend to end of bend, it hasn't any considerable changes.

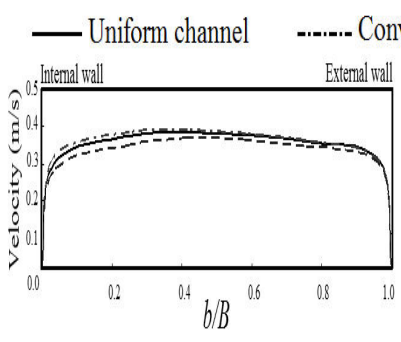

(a) $40^{\circ}$ cross-section

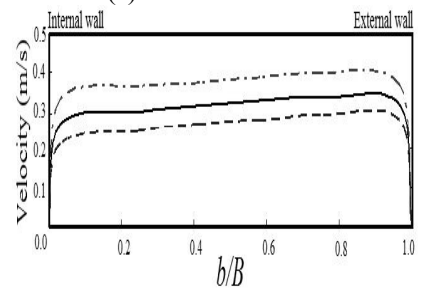

(c) $130^{\circ}$ cross-section

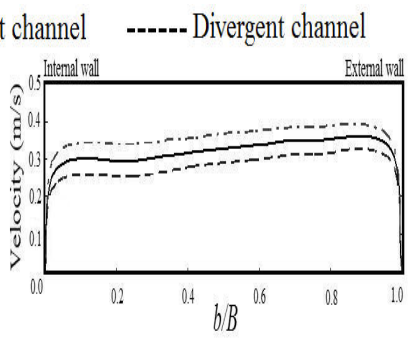

(b) $90^{\circ}$ cross-section

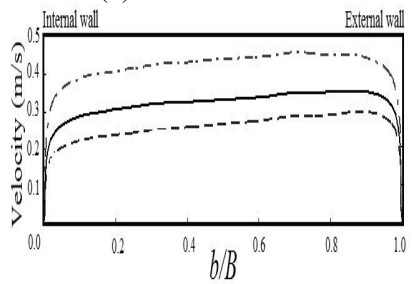

(d) $180^{\circ}$ cross-section
Fig. 6. comparison of velocity profiles at plane near water surface $(\mathrm{h}=0.145 \mathrm{~m})$ for different cross section of uniform, divergent and convergent bends.

TABLE II. COMPARISON PERCENTAGE OF LENGTHWISE VELOCITY DIFFERENCE BETWEEN BENDS AT PLAN NEAR WATER SURFACE

\begin{tabular}{|c|c|c|c|c|c|}
\hline \multicolumn{2}{|c|}{} & \multicolumn{4}{|c|}{ Cross section } \\
\hline \multicolumn{2}{|c|}{ Bend cross section } & $40^{\circ}$ & $90^{\circ}$ & $130^{\circ}$ & $180^{\circ}$ \\
\hline \multirow{2}{*}{ Divergent } & Internal bend & $8 \%$ & $14 \%$ & $19 \%$ & $21 \%$ \\
\cline { 2 - 6 } & External bend & $3 \%$ & $9 \%$ & $12 \%$ & $5 \%$ \\
\hline \multirow{2}{*}{ Convergent } & Internal bend & $10 \%$ & $19 \%$ & $24 \%$ & $27 \%$ \\
\cline { 2 - 6 } & External bend & $7 \%$ & $13 \%$ & $15 \%$ & $19 \%$ \\
\hline
\end{tabular}

Figure 7 shows the path of occurring maximum velocity at the plane near water surface in different bends. As shown, maximum velocity cuts the centerline of channel at about $55^{\circ}$ cross section within uniform bend and this occurs at about $30^{\circ}$ $40^{\circ}$ cross section within convergent channel, while this occurs at about $50^{\circ}$ cross section within divergent channel and its difference with uniform one is the maximum velocity line has a smoother path in the uniform rather than in the divergent bend. This means that in the divergent bend, maximum velocity line cuts the centerline of channel in a straight mood and faster than other bends tangent with external wall of bend in $80^{\circ}$ cross section and stay in this situation to end of bend. In addition, the maximum velocity line is tangent to the external wall of uniform and convergent bend between $90^{\circ}$ to $100^{\circ}$ and stay in this situation to the end of bend. This indicates that in convergent bend, centrifugal force dominates over flow field increases at less distance from the beginning of the bend. In other words, effect of lengthwise pressure gradient dominates to effect of secondary flow strength at a less distance from beginning of the bend, so maximum velocity disposes toward external wall at closer distance from the beginning of the bend.
Moreover, in both cases, maximum velocity becomes tangent to the bend external wall near $100^{\circ}$ cross section and remains in this state until it reaches the end of the bend.

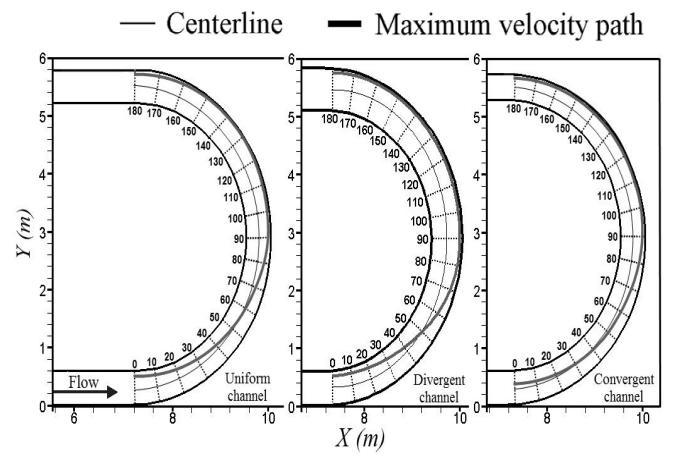

Fig. 7. Comparison of maximum velocity paths at plane of $h=0.145 \mathrm{~m}$ for uniform, divergent and convergent bends.

\section{Comparing helical flow strength}

Helical flow strength is employed to examine the trend of secondary flow dissipation along channels. This concept was defined by Mosonyi and Gotz (1973) as (13) [5]:

$$
i_{S P}=\frac{\int v^{2} d A}{\int u^{2} d A}
$$

Where $\mathrm{u}$ and $\mathrm{v}$ are lengthwise and widthwise velocity components respectively, and $\mathrm{dA}$ is area of each cells. Also, a program is developed for calculating helical flow strength in VB language.

Figure 8 indicates the changes of helical flow strength along bends. Regarding this figure, for three types of channels, the strength of helical flow reaches its maximum value at about $60^{\circ}$ cross section, and this section is the same area where secondary flow dominates lengthwise pressure gradient, resulting in transverse transfer of lengthwise momentum. After secondary flow grows and reaches its perfect growth at $60^{\circ}$ cross section, the strength of helical flow assumes a decreasing trend. Also, because divergent channel lengthwise velocity is lower than the uniform one and lengthwise velocity in uniform channel is lower than the convergent one along the bed, denominator in (13) is longer for channels with convergent bends; as a result the maximum helical flow in divergent, uniform and convergent bend is higher respectively. Also, the maximum of helical flow strength in uniform channel is $10.55 \%$ lower than divergent one.

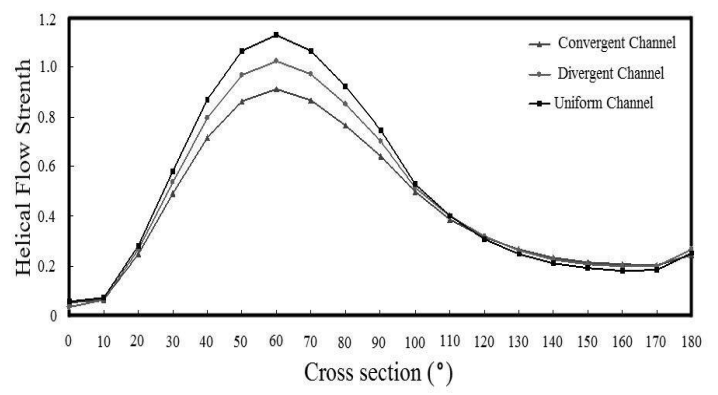

Fig. 8. Changes of helical flow strength along bend at flow rate of $30 \mathrm{~L} / \mathrm{s}$. 


\section{E. Comparing water surface lengthwise and widthwise slopes}

In Figure 9, changes of longitudinal slope of water surface were shown for external wall, center line and internal wall of bends. In all of channels, longitudinal slope of water surface forms before reaching the bend. This phenomenon is caused by the change in lengthwise momentum movement direction due to flow entering into the bend, causing a short interval to be affected before the bend entrance. Then, once the bend is reached, water surface faces relative positive and negative changes at the external wall and the internal wall, respectively, due to the effect of the centrifugal force. In all of channel at about $30^{\circ}$ cross section, the trend of water surface rising at the external wall is positive, then it begins to decrease, and reaches near zero after the curve, in all cases. The water surface varying trends are clearly different for the channels. The range of water surface elevation changes is much wider in the convergent channel than in the uniform channel. More decreasing in water level in convergent channel rather than uniform one is due to effect of narrowness on flow. With decreasing of channel width, the discharge rate per unit of cross section will increase and water level is decreasing considering Specific Energy diagram. Also, water level changes in the uniform and convergent channel is decreasing (negative longitudinal steep) and it's increasing for the divergent channel (positive longitudinal steep). This means that water level will generally decrease in the internal wall, center line and external wall of the uniform and convergent bend when moving forward, but will increase in the divergent one.

Table III shows the decreasing percentage of water level in vicinity of external wall of uniform bend in comparison with divergent bend. As seen, the percentage of water level differences in these two bend will increase with moving from beginning of the bend. Fluctuation changes can be observed on the water surface by careful looking at the flow pattern at the end of uniform bend (the enlarged part in Figure 9), which is caused by the straightening of the bend path. This phenomenon was not observed at the exit of the convergent and divergent bend. It can be seen from Figure 10 that the widthwise elevation difference of the water surface occurs before the beginning of the bend. Such an elevation difference increases after the flow enters the bends and decreases as the flow approaches the exit of the bend, reaching zero shortly after the flow exits the bend.

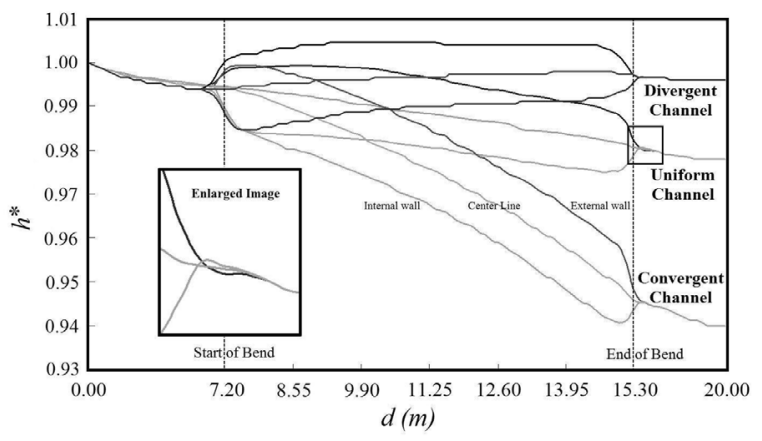

Fig. 9. Trend of lengthwise water surface changes at internal and external walls of uniform, convergent and divergent channels $d=$ distance from the beginning of channel $(\mathrm{m}), \mathrm{h}^{*}=$ dimensionless water depth ratio.
TABLE III. DECREASING PERCENTAGE OF LONGITUDINAL WATER SURFACE LEVEL FOR EXTERNAL WALL OF UNIFORM BEND IN COMPARISON WITH UNIFORM BEND CROSS SECTION

\begin{tabular}{|c|c|}
\hline Decreasing water level (\%) & Bend cross section $\left({ }^{\circ}\right)$ \\
\hline 0.1 & 0 \\
\hline 0.2 & 10 \\
\hline 0.3 & 20 \\
\hline 0.4 & 30 \\
\hline 0.5 & 40 \\
\hline 0.5 & 50 \\
\hline 0.6 & 60 \\
\hline 0.7 & 70 \\
\hline 0.8 & 80 \\
\hline 0.8 & 90 \\
\hline 0.9 & 100 \\
\hline 1.0 & 110 \\
\hline 1.1 & 120 \\
\hline 1.2 & 130 \\
\hline 1.3 & 140 \\
\hline 1.3 & 150 \\
\hline 1.4 & 160 \\
\hline 1.5 & 170 \\
\hline 1.6 & 180 \\
\hline
\end{tabular}

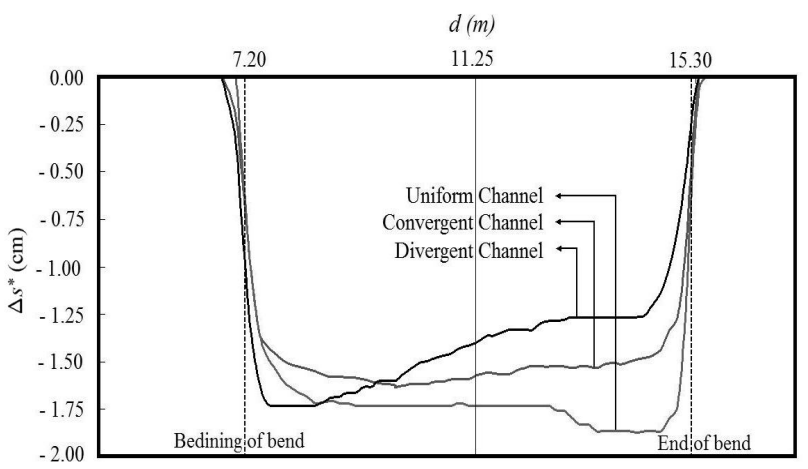

Fig. 10. Lengthwise changes of transverse water surface elevation difference $\left(\mathrm{ds}^{*}\right), \mathrm{d}=$ distance from the beginning of channel $(\mathrm{m})$.

At the beginning of the bend, the widthwise water surface elevation difference is larger in the divergent, uniform and convergent channel, respectively. At the end of the bend, this amount is larger in the uniform, convergent and divergent channel, respectively. Uniform bend have an almost same widthwise water surface elevation difference, from beginning to end of the bend in channel.

\section{F. Comparing flow lines at different levels at flow length}

As can be seen in Figure 11, the pattern of flow lines are almost the same for all of bends but with moving toward the end of divergent bend, flow lines take distance from each other and it shows the decreasing in discharge per unit of width. Opposite of this situation take place in convergent bend. For the all of channels, the path of stream lines moves toward the external wall of the channel at the plane near the water surface. Water particles begin to move from the internal wall of the bend entrance, proceed toward the external wall, and ultimately strike against the channel's external wall downstream. The pattern of stream lines is slightly affected by the secondary flow at this channel's average-depth plane, and nearly follows its curve. At the plane near the bed of the uniform channel, the 
pattern of stream lines excessively deviates toward the internal wall of the bend and water particles do not follow the bend. Therefore, if there were mobile particles on the bed of the bend, it is expected that they would be washed out downstream toward the internal wall.

At the entrance of the bend, the rate of particles' deviation at the plane near the bed is higher than that near the water surface, the reason for which is the presence of one-way flow toward the internal wall at the channel bed as well as the higher intensity of secondary flow at lower flow levels than at upper ones. As can be seen in the channel with a convergent bend, the overall pattern of stream lines is almost identical to that of the channel with the uniform bend, except that in the convergent bend, due to gradual reduction of the channel width and increasing discharge per unit of width, the stream lines move closer together.

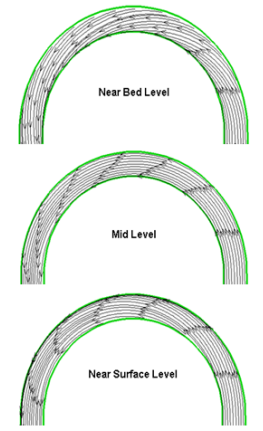

(a) Uniform channel

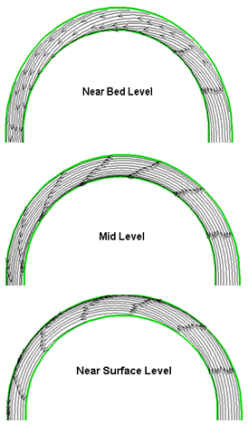

(b) Convergent channel

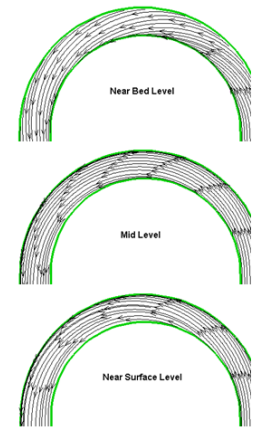

(c)Divergent channel
Fig. 11. Comparison of stream lines in uniform and convergent channels bends.

\section{G. Comparison of bed shear stress distribution}

Although the study of bed variation requires concurrent examination of fluid flow and bed sedimentation as well as their interaction, it is possible to predict erosion and sedimentation patterns for mobile beds by taking the distribution of bed shear stress into account. As can be seen in Figure 12, there is a region of maximum shear stress in both uniform and convergent channels from which mobile bed particles begin to move shortly after the experiment is initiated. The reason for the creation of such a high-stress region is a high-velocity gradient existing here, which is caused by movement of a high-velocity core toward the external wall and its expansion at the plane near the bed. This point can be well interpreted by the changes in the high-velocity core at the $180^{\circ}$ cross-section.

As Figure 12 indicates, at the end of the bend, bed shear stress exhibits higher values in convergent channel and subsequent straight channel than in the same region of channel with uniform bend; the value of maximum bed shear stress is longer in convergent bend than in uniform bend. The reason for that is nothing but the flow impressionability from channel's gradual narrowing. That is, lengthwise flow velocity; hence bed shear stress increase due to this section narrowing.

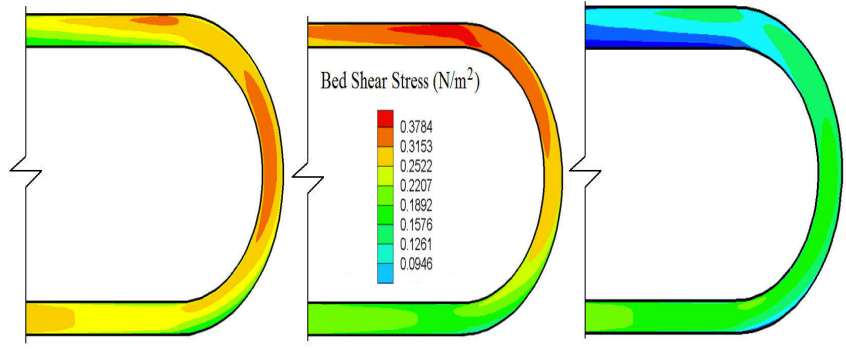

(a) Uniform channel

(b) Convergent channel

(c) Divergent channel

Fig. 12. Comparisons of bed shear stress in uniform and convergent bends for flow rate $30 \mathrm{l} / \mathrm{s}$.

Also, it can be observed that width and length of the region with high bed shear stress are larger at the end of convergent bend than those at the same region of uniform bend. In addition, more attention to distribution of high velocity core at the bottom of channel of this region indicates the mentioned changes in bed shear stress be expected for convergent channels. The region with high bed shear stress which seen at the middle of the uniform bend is not seen in channel with divergent bend. The reason for that is decreasing of velocity due to effect of channel expansion.

\section{CONCLUSION}

In this study, the numerical model was first verified using measured data on the bend with a uniform bend width. Statistical comparison of the calculated and measured velocities at a plane near the water surface shows that the maximum ERMS and EM are equal to $0.074 \mathrm{~m} / \mathrm{s}$ and -0.066 $\mathrm{m} / \mathrm{s}$, respectively, indicating good agreement between measured and calculated velocities in the uniform bend. Flow characteristics and patterns were compared in the uniform, convergent and divergent $180^{\circ}$ bends. Velocity profile shows higher lengthwise velocity at the $45^{\circ}$ and $135^{\circ}$ cross-sections in convergent, uniform and divergent bend, respectively. For the convergent channel, the maximum velocity path crosses the channel centerline near the water surface $(\mathrm{h}=0.145 \mathrm{~m})$ at about the $30^{\circ}$ to $40^{\circ}$ cross-section, while this occurs at about $50^{\circ}$ cross section within divergent channel and about $55^{\circ}$ for uniform one. The varying range of the water surface elevation is much wider for the convergent channel than for the uniform and divergent one. In general, the widthwise water surface elevation difference is larger in the convergent channel than in the uniform one due to the existence of larger centrifugal force in the convergent channel. Also, water level changes in uniform and convergent channel is decreasing (negative longitudinal steep) and it's increasing for divergent channel (positive longitudinal steep). In all of bends, the strength of the helical flow reaches its maximum value at about the $60^{\circ}$ crosssection. The helical flow strength is higher in the divergent, uniform and convergent channel, respectively, and the maximum of helical flow strength in uniform channel is $10.55 \%$ lower than divergent one. Moreover, no counterrotating cell is observed at the $135^{\circ}$ cross-section in convergent channel. In both uniform and convergent channels, a region with maximum bed shear stress is observed at the bend exit, but at the end of the convergent bend, bed shear stress shows higher values than those in the same region in the channel with 
a uniform bend. The pattern of flow lines are almost the same for all of bends but with moving toward the end of divergent bend, flow lines take distance from each other and it shows the decreasing in discharge per unit of width. Opposite of this situation take place in convergent bend.

\section{REFERENCES}

[1] N. Rüther, Computational Fluid Dynamics in Fluvial Sedimentation Engineering. Ph. D. Dissertation, Norwegian University of Science and Technology, Trondheim, Norway, 2006

[2] V. T. Chow, Open Channel Hydraulics, McGraw-Hill, New York, 1959

[3] C. E. Mockmore, "Flow around bends in stable channels", Transactions of the American Society of Civil Engineers, Vol. 109, No. 1, pp. 593618,1994

[4] I. L. Rozovskii, Flow of Water in Bend of Open Channel, Institute of Hydrology and Hydraulic Engineering, Academy of Sciences of the Ukrainian SSR, Kiev, 1957

[5] E. Mosonyi, W. Gotz, Secondary currents in subsequent model bends. Proceedings of the International Association for Hydraulic Research International Symposium on River Mechanics, Asian Institute of Technology, Bangkok, 1973

[6] M. A. Leschziner, W. Rodi, "Calculation of strongly curved open channel flow", Journal of the Hydraulic Division, Vol. 105, No.10, pp. 1297-1314, 1979
[7] H. C. Lien, J. C. Yang, K. C. Yeh, T. Y. Hsieh, "Bend-flow simulation using 2D depth-averaged model", Journal of Hydraulic Engineering, Vol. 125, No. 10, pp. 1097-1108, 1999

[8] R. Booij, "Measurements and large eddy simulations in some curved flumes", Journal of Turbulence, Vol. 4, No. 1, pp. 8-16, 2003

[9] N. R. B. Olsen, A Three-Dimensional Numerical Model for Simulation of Sediment Movements in Water Intakes with Multiblock Option, Department of Hydraulic and Environmental Engineering, Norwegian University of Science and Technology, Trondheim, Norway, 2006

[10] S. L. Huang, Y. F. Jia, S. Y. Wang, "Numerical Modeling of Suspended Sediment Transport in Channel Bends", Journal of Hydrodynamics, Vol. 18, No. 4, pp. 411-417. 2006

[11] L. Begnudelli, A. Valiani, B. F. Sanders, "A balanced treatment of secondary currents, turbulence and dispersion in a depth-integrated hydrodynamic and bed deformation model for channel bends", Journal of Advances in Water Resources, Vol. 33, No. 1, pp. 17-33, 2010

[12] M. Naji Abhari, M. Ghodsian, M. Vaghefi, N. Panahpur, "Experimental and numerical simulation of flow in a $90^{\circ}$ bend", Journal of Flow Measurement and Instrumentation, Vol. 21, No. 3, pp. 292-298, 2010

[13] B. E. Launder, D. B. Spalding, "The numerical computation of turbulent flows", Computer Methods in Applied Mechanics and Engineering, Vol. 3, No. 2, pp. 269-289, 1974

[14] H. Schlichting, Boundary Layer Theory. 7th ed. New York: McGrawHill, 1979

[15] M. Pirestani, Study of Flow and Scouring Patterns at Intake Entrance of Curved Canals, Ph. D. Dissertation, Azad Islamic University, Tehran, Iran, 2004 